\title{
HUMAN ENHANCEMENT: QUESTIONI BIOGIURIDICHE
}

\author{
Lidia Ricci \\ Dipartimento di Scienze Anatomiche \\ Istologiche, Medico Legali e dell'Apparato Locomotore \\ Università Sapienza Roma \\ lidiaricci90@gmail.com \\ Mariangela Cersosimo \\ Ricercatore \\ Università Telematica Internazionale Uninettuno Roma \\ Pasquale Ricci \\ Dipartimento di Scienze Anatomiche \\ Istologiche, Medico Legali e dell'Apparato Locomotore \\ Università Sapienza Roma
}

Fecha de Recepción: 16 Marzo 2019

Fecha de Admisión: 30 Abril 2019

\section{SOMMARIO}

Background: Il potenziamento umano consiste in una modifica volta a migliorare caratteristiche e capacità umane attraverso interventi scientifici o tecnologici sul corpo umano, partendo da una "condizione di integrità" e non da uno stato di malattia. Occorre pertanto valutare se il potenziamento sia lesivo della dignità umana oppure strumento utile a migliorare le condizioni di vita dell'uomo. Metodi: In un primo momento si è proceduto alla ricerca bibliografica dedicata all'inquadramento dello HE mediante l'analisi dei concetti di salute e potenziamento, affrontando gli aspetti di pertinenza biogiuridica come ad esempio i possibili impatti negativi su dignità, identità, libertà ed uguaglianza, e mettendo a confronto teorie scientifiche ed umanistiche. In un secondo momento si è proceduto all'analisi dettagliata del quadro normativo nazionale e sovranazionale degli ultimi dieci anni, dallo Human Enhancement Study dello Science and Technology Options Assessment ai pareri del Comitato Nazionale per la Bioetica. A partire da tale inquadramento sono state individuate e trattate alcune tipologie di potenziamento, utilizzando un approccio metodologico medico sociale ed approfondendo ciascuna tematica sotto il profilo sia dottrinario sia giurisprudenziale. Risultati: Doping, chirurgia estetica e divieto di selezione eugenetica sono tecniche già disciplinate. Smart drugs e deep brain stimulation sono tecniche già disciplinate per utilizzo a scopo terapeutico, ma talvolta il loro utilizzo esula dalla cura di patologie. Potenziamento militare e Potenziamento biologico sono tecniche in fase sperimentale. Di ciascuna di esse sono state rappresentate le peculiari diffi- 
coltà nell'identificazione dei limiti di liceità. Conclusioni: Sembrerebbe ipotizzabile un approccio "case by case" del diritto, poiché duttile ed in grado di adattarsi alle caratteristiche peculiari di ogni singola forma di potenziamento valutandone al contempo l'impatto politico, legale, etico, sociale e di sicurezza, in un quadro di disomogeneità delle Tecnologie di Potenziamento Umano che non permette una cornice normativa unitaria in grado di soddisfare pienamente i bisogni individuali e collettivi.

Parole chiave: classificazione tipologie potenziamento umano; tecnologie di potenziamento umano; quadro normativo; problematiche biogiuridiche

\section{ABSTRACT}

Human enhancement: bio-juridical issues. Background: Human Enhancement is a modification aimed to improve human's characteristics and capabilities through scientific or technological interventions in the human body, starting from an 'integrity condition' and not from a state of disease. Therefore, we need to value if the enhancement is harmful of the human dignity or if it's an useful tool to improve the human life's condition. Methods: At first we focused on the bibliographic research dedicated to the HE's classification through the analysis of health's and enhancement's definitions, confronting with the bio-juridical perspective as for example the possible negative effects over a person's dignity, identity, freedom, equality; and comparing scientific and humanistic theories. We later examined in detail the national and the supranational legal framework of the last ten years, from the Science and Technology Options Assessment's Human Enhancement Study to the Comitato Nazionale per la Bioetica's opinions. Starting from this classification we located and analyzed some typologies of enhancement, using a medical social methodological approach and deepening each subject matter from both doctrinaire and jurisprudential profile. Results: Doping, cosmetic surgery and prenatal genetic enhancement's prohibition are techniques already disciplined. Smart drugs and deep brain stimulation are techniques already disciplined for therapeutic purpose, but sometimes their application lies outside the disease's therapy. Military and biological enhancement are techniques in an experimental phase. We have represented the particular difficulties in identifying the limits of lawfulness of each of these typologies of enhancement. Conclusions: We could hypothesize a 'case by case' law's approach, because it's ductile and able to adapt on the peculiar characteristics of every single form of enhancement, evaluating at the same time political, legal, ethical, social and safety impact, in a context of inhomogeneity of the Human Enhancement Technologies that it doesn't allow an uniform normative framework able to fully satisfy individual and collective needs.

Keywords: classification of human empowerment types; human enhancement technologies; regulatory framework; bio-legal issues

II potenziamento umano consiste in una modifica volta a migliorare la performance umana individuale attraverso interventi scientifici o tecnologici sul corpo umano ${ }^{1}$. Esso si basa su tecniche per migliorare la conoscenza, per trasformare l'aspetto estetico, per aumentare prestazioni sportive, fisiche, intellettuali, aumentare capacità di lavoro ${ }^{2}$. Si è di fronte ad un "fenomeno" utilizzato per migliorare un funzionamento considerato già nel "range" della normalità ${ }^{3}$. Sono dunque da considerare tecnologie di Human Enhancement quelle che vengono utilizzate non per trattare la malattia, ma per migliorare le caratteristiche e le capacità umane. Si tratta di finalità che eccedono il caratteristico concetto di cura, anche se la linea di demarcazione tra potenziamento e terapia è labile. Lo Science and Technology Options Assessment (STOA), al riguardo, nello "Human Enhancement Study" ha 
tentato di delimitarne il campo tra tre ordini di intervento. II non potenziamento, ossia il trattamento di una malattia, ed è questa la medicina tradizionale; il Potenziamento terapeutico, ossia il trattamento di una malattia con effetti che eccedono il ripristino dello stato di salute iniziale, quindi si va oltre la cura tradizionale; il Potenziamento non terapeutico, ossia il trattamento volto a migliorare un funzionamento considerato già nel "range" della normalità 4 .

Sin dal principio l'utilizzo del progresso della scienza e delle tecnologie bioetiche per fini lontani dalla tradizionale terapia ha suscitato non poche perplessità, generando un vero e proprio contrasto etico-filosofico. I maggiori obiettori del potenziamento sono i Bioconservatori, i quali ritengono che il tanto ricercato miglioramento dell'uomo possa in realtà provocare una deumanizzazione, apportando cambiamenti talmente profondi da alterare la specie in modo radicale ${ }^{5}$. Inoltre sottolineano l'immoralità celata in tali tecniche qual è quella di "giocare a fare Dio". D'altro canto, sul filone opposto, i Transumanisti sono i maggiori sostenitori del potenziamento, accolto con entusiasmo non solo per il benessere individuale ma anche per quello collettivo. Infatti, grazie ad esso si potrebbero eliminare tutti gli aspetti più fragili ed indesiderati della specie umana, quali la malattia, l'invecchiamento, le debolezze fisiche ed intellettuali, e controllare lo stato mentale e l'umore 6 . Ad oggi il problema morale ed etico maggiormente sollevato risulta essere l'impatto che le tecnologie di potenziamento potrebbero avere sull'autenticità dell'uomo, intesa come identificazione dell'individuo con le proprie capacità e caratteristiche fondamentali che verrebbero alterate attraverso interventi non rispondenti ad esigenze mediche. Nonostante le polemiche e le problematiche sottese, bisogna tuttavia considerare il potenziamento come un fenomeno inevitabile poiché il cambiamento è insito in natura ed è ragione principale della nostra evoluzione. Infatti tali tecnologie potrebbero al tempo stesso anche apportare reali miglioramenti per il singolo e, più in generale, potrebbero contribuire alla realizzazione di un mondo migliore. Occorre perciò valutarne pro e contro mediante un'analisi dei singoli casi, sulla base di una valutazione rischi-benefici, effettuando dunque un bilanciamento di interessi.

A tal fine sono state trattate sette tipologie di potenziamento (doping, chirurgia estetica, divieto di selezione eugenetica, smart drugs, deep brain stimulation, potenziamento militare e potenziamento biologico), approfondendo ciascuna tematica sia sotto il profilo dottrinario sia sotto il profilo giurisprudenziale, ed affrontando nello specifico gli aspetti di pertinenza biogiuridica, prendendo le mosse dal concetto di salute (diritto fondamentale - art. 32 Cost.), dalla Dichiarazione Universale dei diritti dell'Uomo, dalla Carta dei diritti fondamentali dell'Unione Europea, dalla Dichiarazione Universale sulla Bioetica e i Diritti Umani dell'UNESCO. Queste tipologie sono state raggruppate in tre categorie: tecniche già disciplinate giuridicamente; farmaci o trattamenti normalmente utilizzati a scopo terapeutico e a tal fine giuridicamente regolati, utilizzati a scopi potenziativi; tecniche in fase sperimentale. Le tipologie trattate rientrano tutte nelle più ampie forme di enhancement: cognitive, physical, genetic Enhancement.

Nella prima categoria vi rientrano il doping, la chirurgia estetica ed il divieto di selezione eugenetica. II doping consiste nell'uso di sostanze e metodi atti ad alterare artificialmente le condizioni fisiche e/o biologiche dell'atleta7. II Codice Mondiale Antidoping (World Anti-Doping Agency 2003) ha introdotto una Prohibited List ed una responsabilità oggettiva per l'atleta che risulti positivo ai controlli antidoping. La responsabilità oggettiva sarà sempre assoluta, ossia senza ammissione di prova liberatoria, con conseguente annullamento dei risultati della singola competizione, in quanto l'atleta in ogni caso ha ottenuto un vantaggio illecito rispetto agli altri. In aggiunta tale responsabilità oggettiva sarà relativa, ossia con ammissione di prova liberatoria, in relazione alla squalifica da tutte competizioni, per un periodo più o meno lungo ${ }^{\circ}$. Obiettivo di tale Codice non è solo quello di tutelare la salute degli atleti, ma anche di tutelare l'uguaglianza nelle competizioni e l'etica sportiva. 
Infatti nella lista proibita vengono inserite anche sostanze non nocive per la salute, o comunque di cui non si ha certezza di pericolosità 9 . Obiettivo quest'ultimo espressamente individuato dalla Convenzione Internazionale contro il Doping nello Sport (The United Nations Educational, Scientific and Cultural Organization 2005) che esplicita nel suo Preambolo il principio della correttezza a salvaguardia delle condizioni di uguale partecipazione alle gare, con l'obiettivo di evitare prestazioni sportive artificiose. In Italia la L.2000, N. 376 ("Disciplina della tutela sanitaria delle attività sportive e della lotta contro il doping") descrive il ruolo dell'attività sportiva in chiave promotrice della salute individuale e collettiva ${ }^{10}$. Dunque tutela primaria ed obiettivo fondamentale è proprio la salute. La selezione eugenetica prenatale consiste nella selezione delle persone sulla base delle caratteristiche genetiche considerate migliori, effettuata su embrioni prodotti in vitro o feti in utero. Problema principale è la violazione dei diritti fondamentali della persona, e più nello specifico della pari dignità interindividuale ${ }^{11}$. Sul punto è stato espresso un divieto assoluto, a partire dalla Dichiarazione Universale dei Diritti dell'Uomo 1948 e dalla Convenzione Europea per la salvaguardia dei Diritti dell'uomo 1950 che, tutelando espressamente la pari dignità, hanno indirettamente manifestato il divieto alla selezione eugenetica prenatale ${ }^{12}$. In maniera più esplicita, invece, il divieto è sancito dalla Carta dei Diritti Fondamentali dell'Unione Europea 2000: "(...) divieto delle pratiche eugenetiche, in particolare di quelle aventi come scopo la selezione delle persone"13. Anche in Italia vi fu un divieto esplicito con L. 2004 N. 40 ("Norme in materia di procreazione medicalmente assistita") relativamente alla "diagnosi genetica pre-impianto" ed alla selezione (eventuale) degli embrioni risultati non anomali (art. 13), in quanto ritenuta "pratica di selezione eugenetica sugli embrioni"14,15. Tuttavia con la sent. 229/2015 della Corte Costituzionale è stata dichiarata l'illegittimità dell'art. 13 L. 40/2004 nella parte in cui contempla come ipotesi di reato anche gli interventi aventi finalità diagnostiche $\mathrm{e}$ terapeutiche, sulla base del presupposto che se si ammette l'aborto in caso di gravidanza seriamente pericolosa per la salute della donna, non ammettere la possibilità di evitare l'impianto di embrioni non sani integrerebbe una violazione degli articoli 3 e 32 Cost. ${ }^{16,17}$. Infine la chirurgia estetica consiste in tutti quegli interventi di modificazione di parti del proprio corpo per esigenze soggettive di adeguamento ad un ideale corporeo. Si differenzia pertanto dalla chirurgia ricostruttiva, in cui esigenza estetica e terapeutica si sovrappongono. Considerando che l'Organizzazione Mondiale della Sanità ha esteso il concetto di salute intendendolo come benessere psichico e sociale (e non più solo come assenza da patologie fisiche $)^{18}$, oggi l'intervento chirurgico estetico rientrerebbe nei trattamenti terapeutici laddove sia a fondamento dell'identità dell'individuo in funzione delle sue relazioni sociali ${ }^{19}$. Ed è proprio quanto richiamato in ambito Nazionale superando così il problema degli atti di disposizione del proprio corpo di cui all'art. 5 Cod. Civ. ${ }^{20}$. Infatti il Comitato Nazionale di Bioetica prevede che la richiesta di modificazione del corpo non può prescindere da un riferimento, diretto 0 indiretto, alla dimensione terapeutica. I medici a tal fine devono usare criteri di proporzionalità e accuratezza, effettuando sempre una valutazione rischi-benefici, verificando le condizioni fisiche e psichiche del paziente, e dando una esaustiva informazione relativamente all'intervento, al fine di evitare lo sfruttamento del corpo 21 .

Nella seconda categoria vi rientrano le smart drugs e la deep brain stimulation. Le Smart Drugs (cd. droghe intelligenti) sono quei Farmaci di ultima generazione che agiscono direttamente sul sistema nervoso centrale e sul cervello, incrementando prestazioni mnemoniche o cognitive (es capacità di attenzione e concentrazione $)^{22}$. Vengono utilizzati, per esempio, su soggetti affetti dal morbo di Alzheimer o ADHD (deficit dell'attenzione), ma sono farmaci utilizzati spesso anche da soggetti over50 (e non solo!) al fine di potenziare memoria ed apprendimento (es Metilfenidato). Mentre per i primi si conoscono scientificamente conseguenze e benefici, per i secondi no. Le perplessità che sorgono, dunque, riguardano l'effettiva capacità di potenziamento della performance di 
soggetti sani, ed in caso positivo sorge il dubbio se lo sviluppo "artificiale" di certe capacità possa interferire con l'ordinario esercizio di quelle naturali. Partendo dal presupposto che ad oggi non esiste una normativa di tutela della mente (si ricorre piuttosto al diritto di pensiero e di integrità mentale), I'Unione Europea ha dato vita ad una Strategia dell'Unione Europea in materia di Droga (2012/C 402/01) basata sulla cooperazione internazionale in vista di una strategia integrata ed equilibrata di lotta contro la droga, la quale è anche volta a fornire valore aggiunto alle singole strategie naziona${ }_{1 i}{ }^{23}$. In ambito Nazionale l'Istituto Superiore di Sanità nel libro "Smart Drugs" individua le più comuni e rischiose smart drugs, ma senza pretesa di completezza considerando anche che la definizione stessa di smart drugs è in continuo cambiamento ${ }^{24}$. E' importante sottolineare come in Italia nel 1993 la modifica al D.P.R. n. 309 del 1990 ha portato alla depenalizzazione della detenzione ed assunzione di sostanze stupefacenti (anche psicotrope, per le quali però resta necessaria la prescrizione medica) per uso personale 25 . Invece la Deep Brain Stimulation è una tecnica personalizzata con effetti reversibili, consistente nell'impianto di elettrodi in alcune regioni cerebrali mediante operazione chirurgica. Ad oggi tale tecnica è ammessa solo per alcune patologie (es. Parkinson e malattie che causano tremore), per le quali è prevista in ogni caso una ferrea regolamentazione, a causa delle pesanti conseguenze psico-fisiche legate all'intervento: possono accedervi solo soggetti che si trovano in circostanze estreme di particolare sofferenza, qualora non vi siano tecniche alternative di intervento. Non è, pertanto, tecnica utilizzata a scopi di potenziamento, ma la possibilità che in un futuro possa aprirsi un varco in tal senso desta particolari preoccupazioni. In particolar modo si canalizza l'attenzione sulla possibilità di una "manipolazione della mente e della memoria" fino ad arrivare a poter "accendere e spegnere a piacimento" le emozioni e gli stati d'animo come se ci fosse un interruttore 26 . In sostanza si apre la possibilità di scegliere quali emozioni e sensazioni provare. Tutto ciò scalfisce il concetto di personalità, inteso non solo come identificazione fisica della persona, ma anche (e forse soprattutto) come ciò che una persona è a livello caratteriale ed emotivo. Si dovrebbe dunque riflettere sul grado in cui un soggetto rimarrebbe se stesso, con particolare attenzione alla tutela della integrità e della dignità.

Nella terza ed ultima categoria rientrano il potenziamento militare ed il potenziamento biologico. Anche se si tratta di tecnologie in fase sperimentale, si può analizzare una posizione primordiale allo stato odierno della sperimentazione. II Potenziamento Militare consiste in tecniche innovative volte al superamento delle normali capacità umane al fine di aumentare il "quoziente di difesa/offesa". Al riguardo bisogna anzitutto distinguere tra ottimizzazione della performance umana e potenziamento della performance umana. La prima consiste in strategie volte a sostenere la performance del soldato davanti a fattori di stress che la mettono a rischio, intese in chiave di "aiuto/sostegno" al soldato (es. addestramento fisico e psicologico a determinate situazioni, controllo fisico, emozionale e mentale dello stress e di altre circostanze difficili). II secondo, invece, consiste in strategie per creare capacità umane che vanno oltre la normale variabilità biologica attraverso la modificazione della funziona umana: modifiche genetiche e farmacologiche, stimolazione neuronale. Dubbie sono, però, le probabili ricadute sull'equilibrio psico-fisico del soldato; e talvolta si pone il problema di un soldato inteso come "macchina da guerra" e non più persona (in tal caso si può addirittura arrivare a riconoscerlo come arma biologica, ed in quanto tale vietata dalla Convezione sulle armi biologiche e tossiniche del $\left.1975^{27}\right)^{28}$. In via sperimentale il potenziamento militare sembrerebbe essere l'approccio utilizzato in America (US Defense Advanced Research Projects Agency) attraverso quattro forme di potenziamento: fisico, cognitivo, dei sensi e metabolico ${ }^{29}$. In Italia invece il Comitato Nazionale di Bioetica individua la tutela della vita e della salute, della dignità e dell'integrità come carattere preminente nei confronti di qualsiasi esigenza, sottolineando che il "dovere di obbedienza" del soldato non legittima in alcun modo la violazione di tali diritti 30 . In Italia, quindi, si sposa l'idea 
di ottimizzazione della performance umana. Infine il Potenziamento Biologico consiste nel tentativo di controllare, fino ad annullare, la degenerazione progressiva di cellule, tessuti ed organi, per evitare l'invecchiamento fino ad eliminare del tutto la morte (ad oggi mera utopia). I sostenitori di tale potenziamento ritengono che vita e salute siano beni primari ed in quanto tali devono essere tutelati in qualsiasi modo. Gli oppositori, invece, ritengono che la morte possa addirittura essere un bene per l'uomo, in quanto la finitudine è vista come una spinta per l'uomo verso aspirazioni, impegno e passioni. Le ricerche sul potenziamento biologico si sviluppano in tre campi: impiego di ormoni, per un rinvigorimento fisico; ricerca su interruttori genetici, i quali regolano il processo biologico dell'invecchiamento; ricerca su cellule staminali (adulte ed embrionali), per garantire un rifornimento illimitato di tessuti ed organi sostitutivi ${ }^{31}$. E' proprio su quest'ultimo campo sperimentale, e più in particolare sulla ricerca su cellule staminali embrionali, che l'Europa si vede spaccata in due tra paesi sostenitori, che ammettono la sperimentazione sugli embrioni (seppur con regolamentazione ferrea per evitare strumentalizzazione di embrioni e sperimentazioni arbitrarie) ritenendo che l'embrione sia vita solo nel momento in cui venga impiantato in utero ${ }^{32}$; e paesi oppositori, che vietano la sperimentazione sugli embrioni ritenendo che la cellula uovo sia già vita. Tra questi ultimi s'inserisce I'Italia che vieta tali sperimentazioni con la L.40/2004, eccetto che per scopi terapeutici (come già analizzato) ${ }^{33}$.

\section{CONCLUSIONI}

Data la Disomogeneità delle Tecniche di Human Enhancement sembrerebbe più probabile ipotizzare un approccio "case by case" del diritto, in quanto in grado di adattarsi alle caratteristiche peculiari di ogni singola forma di potenziamento 34 . Dalla dissertazione fin qui condotta infatti, ad oggi in Italia risulta essere stato adottato tale approccio. Tuttavia sembrerebbe più opportuno tentare di individuare un punto di incontro tra le varie tecnologie, al fine di permettere una cornice normativa unitaria in grado di soddisfare pienamente i bisogni individuali e collettivi che sia, al tempo stesso, al passo con l'evoluzione sociale e scientifica. A tal fine, data l'analisi svolta fin qui, sono stati individuati come comuni beni fondamentali ed imprescindibili, possibili oggetto di tutela di una ipotetica normativa comune, la salute, la sicurezza sociale, ed i costi economici gravanti sulla collettività, considerando sempre necessario un bilanciamento tra quelli che sono i diritti e gli interessi del singolo, con i diritti e gli interessi della collettività.

\section{BIBLIOGRAFIA}

Amato, S. (2014). Neuroscienze e utilizzazione militare delle ricerche di potenziamento umano. Etica \& Politica, 16, 182-198.

Bilancetti, M., \& Bilancetti, F. (2013). La responsabilità penale e civile del medico: Casistica aggiornata, approfondimenti giurisprudenziali - profili deontologici (8th ed.). Padova: CEDAM.

Bostrom, N. (2005). In defense of posthuman dignity. Boiethics, 19, 202-214.

Carta dei diritti fondamentali dell'Unione Europea. (2012). Gazzetta ufficiale dell'Unione Europea, C $326 / 02$.

Comitato Nazionale per la Bioetica. (2012). Aspetti bioetici della chirurgia estetica e ricostruttiva. Roma: Author.

Comitato Nazionale per la Bioetica. (2013). Diritti umani, etica medica e tecnologie di potenziamento (enhancement) in ambito militare. Roma: Author.

Corte Costituzionale. Sentenza 11 Novembre 2015, n. 229. Gazzetta Ufficiale n. 46 del 18 Novembre 2015.

D’Amico, M., \& Liberali, B. (2016). Procreazione medicalmente assistita e interruzione volontaria 
della gravidanza: problematiche applicative e prospettive future. Napoli: Edizioni Scientifiche Italiane.

Dipartimento del farmaco - Osservatorio Fumo Alcol e Droga Istituto Superiore di Sanità-. (2015). Smart Drugs (3rd ed.). Roma: Author.

Donati, L., Farneti, A., Gualdi, G., Mangili, F., Pajardi, P., Pennasilico, M., Pennasilico, E., \& Pozzato, R. (1988). Chirurgia plastica ricostruttiva e chirurgia estetica: Aspetti etici, giuridici e medicolegali. Milano: Giuffrè.

Eronia, 0. (2012). Doping mentale e concetto di salute: una possibile regolamentazione legislativa? Archivio Penale,3, 1-23.

European Parliament. (2009). Human Enhancement Study. Brussels: STOA-Science and Technology Options Assessment.

Greppi, E., \& Vellano, M. (Eds). (2010) Diritto internazionale dello sport (2nd ed.). Torino: Giappichelli.

Legge 14 Dicembre 2000 n. 376, Disciplina della tutela sanitaria delle attività sportive e della lotta contro il doping. Gazzetta Ufficiale n. 294 del 18 dicembre 2000.

Legge 19 febbraio 2004 n. 40, Norme in materia di procreazione medicalmente assistita. Gazzetta Ufficiale n. 45 del 24 febbraio 2004.

Lin, P., Mehlman, M.J.M., \& Abney, K. (2013). Enhanced Warfighters: Risk, Ethics, and Policy. The Greenwall Foundation.

Macip, S. (2016). E' possibile frenare l'invecchiamento?: La scienza alle frontiere della vita. Scoprire la scienza, 32. Hachette Fascicoli.

Norme Sportive Antidoping: Documento tecnico-attuativo del Codice Mondiale Antidoping WADA e dei relativi Standard internazionali. (2018). Nado Italia, 1.

Palazzani, L. (2015). Il Potenziamento Umano, tecnoscienza, etica e diritto. Torino: Giappichelli.

Quaranta, G. (Ed.). (2014). I/ doping della mente: Le sfide del potenziamento cognitivo farmacologico. Padova: Edizioni Messaggero.

Ricci, G., \& Fedeli, P. (2004). La chirurgia estetica tra percezione sociale e modello etico deontologico. Difesa sociale,2, 115-132.

Ricci, L., Di Nicolò, B., Ricci, P., Massoni, F., \& Ricci, S. (2019). L'esercizio del diritto al di là della terapia: Io human enhancement. BioLaw Journal,1, 497-512.

Sgreccia, E., \& Tarantino, A. (Eds). (2010). Enciclopedia di Bioetica e Scienza Giuridica: Cadaverecyborg. Napoli, Roma: Edizioni Scientifiche Italiane.

Sgreccia, E., \& Tarantino, A. (Eds). (2012). Enciclopedia di Bioetica e Scienza Giuridica: Ebraismoevoluzionismo. Napoli, Roma: Edizioni Scientifiche Italiane.

Strategia dell'Unione Europea in materia di droga (2013-2020). (2012). Gazzetta ufficiale dell'Unione Europea, C 402/01.

Università degli Studi di Palermo (2014). Enhancement Technologies e diritto penale. Palermo: Università degli Studi di Palermo.

1 European Parliament, Science and Technology Options Assessment (STOA), C. Coenen, M. Schuijff, M. Smits, P. Klaassen, L. Hennen, M. Rader, G. Wolbring, Human Enhancement Study, 2009: 22.

2 L. Palazzani // Potenziamento Umano, tecno scienza, etica e diritto, Torino 2015: 71-72.

3 L. Palazzani, I/ Potenziamento Umano, tecno scienza, etica e diritto, Torino 2015: 6. 
4 European Parliament, Science and Technology Options Assessment (STOA), C. Coenen, M. Schuijff, M. Smits, P. Klaassen, L. Hennen, M. Rader, G. Wolbring, Human Enhancement Study, 2009: 19-20.

5 Nick Bostrom. In defense of posthuman dignity. In Boiethics 2005;19: 202-214.

6 Ibidem

7 Art. 1 L. 14 Dicembre 2000, n. 376, in materia di "Disciplina della tutela sanitaria delle attività sportive e della lotta contro il doping", pubblicata nella Gazzetta Ufficiale n. 294 del 18 dicembre 2000.

8 Art. 4 Norme Sportive Antidoping (NSA) 2018.

9 S. Bastianon, F. Bestagno, S. M. Carbone, M. Coccia, A. Comba, C. Dordi, L. Fumagalli, C. Garbarino, E. Greppi, B. Nascimbene, R. Sapienza, F. Seatzu, M. Vellano, a cura di Edoardo Greppi e Michele Vellano, Diritto internazionale dello sport, seconda edizione, Torino, 2010: 207ss.

10 L. 14 dicembre 2000, n. 376, in materia di "Disciplina della tutela sanitaria delle attività sportive e della lotta contro il doping", pubblicata nella Gazzetta Ufficiale n. 294 del 18 dicembre 2000.

11 Enciclopedia di Bioetica e Scienza Giuridica, vol. V, Edizioni Scientifiche Italiane, 2012: 889-890.

12 L. Ricci, B. Di Nicolò, P. Ricci, F. Massoni, S. Ricci, L'esercizio del diritto al di là della terapia: Io human enhancement. BioLaw Journal- Riv. di Biodiritto, 2019;1:502-504.

13 Art. 3 co. 2 lett. c) Carta dei diritti fondamentali dell'Unione Europea.

14 Art. 13 L. 19 febbraio 2004, num. 40, "Norme in materia di procreazione medicalmente assistita", pubblicata nella Gazzetta Ufficiale n. 45 del 24 febbraio 2004.

15 M. D’Amico, B. Liberali, Procreazione medicalmente assistita e interruzione volontaria della gravidanza: problematiche applicative e prospettive future, Edizioni Scientifiche Italiane, Napoli 2016: 99-103.

16 Corte Costituzionale, sent. n. 229 del 2015, pubblicata in G. U. 18/11/2015 n. 46.

17 M. D’Amico, B. Liberali, Procreazione medicalmente assistita e interruzione volontaria della gravidanza: problematiche applicative e prospettive future, Edizioni Scientifiche Italiane, Napoli 2016: $115-116$ e 121-125.

18 G. Ricci, P. Fedeli, La chirurgia estetica tra percezione sociale e modello etico deontologico, in Difesa sociale, 2004: 116.

19 E. Pennasilico, Liceità giuridica ed etica professionale, in AA.VV., Chirurgia plastica ricostruttiva e chirurgia estetica, Milano: Giuffrè Editore, 1988: 78.

20 M. Bilancetti, F. Bilancetti, La responsabilità penale e civile del medico. Casistica aggiornata, approfondimenti giurisprudenziali - profili deontologici, ottava edizione, Padova, 2013: 652.

21 Comitato Nazionale per la Bioetica, Aspetti bioetici della chirurgia estetica e ricostruttiva: 8.

22 M. Reichlin, P. Benanti, II doping della mente. Le sfide del potenziamento cognitivo farmacologico: 9.

23 Strategia dell'Unione Europea in materia di droga 2013-2020 (2012/C 402/01), art. 3 Prefazione.

24 Dipartimento del Farmaco- Istituto Superiore di Sanità, Smart Drugs, terza edizione, 2015.

25 0. Eronia, Doping mentale e concetto di salute: una possibile regolamentazione legislativa?, in Archivio Penale, 2012;3: 13.

26 0. Eronia, Enhancement Technologies e diritto penale, Università degli Studi di Palermo: 2014. (tesi di dottorato di ricerca in fondamenti del diritto europeo e metodologia comparatistica, anno accademico 2008/2009: 32).

27 S. Amato, Neuroscienze e utilizzazione militare delle ricerche di potenziamento umano, in Etica \& Politica, Vol. XVI, 2014:192-195. 
28 Comitato Nazionale per la Bioetica, Diritti umani, etica medica e tecnologie di potenziamento (enhancement) in ambito militare, 22 febbraio 2016: 16-17.

29 P. Lin, M. J. M. Mehlman, K. Abney, The Greenwall Foundation, Enhanced Warfighters: Risk, Ethics, and Policy, 2013: 21ss.

30 Comitato Nazionale per la Bioetica, Diritti umani, etica medica e tecnologie di potenziamento (enhancement) in ambito militare, 22 febbraio 2016: 15-17.

31 S. Macip, E' possibile frenare l'invecchiamento? La scienza alle frontiere della vita, Hachette, 2016: 81-84.

32 Enciclopedia di Bioetica e Scienza Giuridica, vol. III, Napoli, 2010: 137ss.

33 Legge 19 febbraio 2004, n. 40, "Norme in materia di procreazione medicalmente assistita", pubblicata nella Gazzetta Ufficiale n. 45 del 24 febbraio 2004.

34 L. Ricci, B. Di Nicolò, P. Ricci, F. Massoni, S. Ricci, L'esercizio del diritto al di là della terapia: 10 human enhancement. BioLaw Journal- Riv. di Biodiritto, 2019;1: 512. 
